\title{
Analysis of aerodynamic sound noise generated by a large-scaled wind turbine and its physiological evaluation
}

\author{
T. Inagaki $\cdot$ Y. Li $\cdot$ Y. Nishi
}

Received: 23 June 2013/Revised: 4 March 2014/Accepted: 27 March 2014 / Published online: 10 April 2014

(C) Islamic Azad University (IAU) 2014

\begin{abstract}
Aerodynamic noise generated from a modern large-scale wind turbine was measured and analyzed from an engineering point of view. The measurement items were the sound, the sound pressure level (including the infrasound with extremely low-frequency band) and the corresponding physiological evaluation. Fifteen test subjects received various sound stimuli, including the recorded aerodynamic noise and a synthetic periodical sound, were examined with an electroencephalogram as a physiological evaluation. It was observed from the mapping patterns of brain waves that alpha $a_{1}$ rhythm, which indicates a relaxed and concentrated state, after the sound stimulus with the frequency band of $20 \mathrm{~Hz}$, showed the lowest value among the other cases. That is, the test subjects cannot keep relaxed and their concentration after hearing the sound stimulus at the frequency band of $20 \mathrm{~Hz}$. The induced rate of $a l p h a_{1}$ rhythm almost decreased when the test subjects listened to all the sound stimuli, and further decreased with decreased frequency. Meanwhile, beta $_{1}$ rhythm, which shows a strain state, after the sound stimulus with the frequency band of $20 \mathrm{~Hz}$, showed the highest value among the other cases. Therefore, the infrasound (e.g., low frequency and inaudible for human hearing) was considered to be an annoyance to the technicians who work in close proximity to a modern large-scale wind turbine.
\end{abstract}

Keywords Infrasound noise - Aerodynamic noise - Wind turbine - Environmental impact assessment .

Electroencephalogram $\cdot$ Physiological evaluation

T. Inagaki · Y. Li $(\bowtie) \cdot$ Y. Nishi

Department of Mechanical Engineering, College of Engineering,

Ibaraki University, 4-12-1 Nakanarusawa, Hitachi-shi,

Ibaraki-ken 316-8511, Japan

e-mail: yanrong@mx.ibaraki.ac.jp

\section{Introduction}

Wind energy is one of the alternatives to other energy sources based on fossil fuels, because it is clean, renewable and does not emit any greenhouse gas, such as carbon dioxide or methane (Jowder 2009; Tascikaraoglu et al. 2011; Baath 2013). Recently, more and more investigations were conducted to optimize the performance of wind turbines (Ghoshal et al. 2000; Nagai et al. 2009; Pratumnopharat et al. 2013; Yang and Sun 2013; Yen and Ahmed 2013). For instance, a method to control the pitch angle of turbines to maximize the aerodynamic power of a wind farm was proposed (Lee et al. 2013a, b). An initial design study of a novel concept for tri-axial testing of large wind turbine blades was conducted (Malhotra et al. 2012). Effect of blade flutter and electrical loading on small wind turbine noise was estimated (Vick and Broneske 2013). Structure, airfoil and design optimization of wind turbine blade was investigated (Göçmen and Özerdem 2012; Chen et al. 2013; Lee et al. 2013a, b). Moreover, some numerical studies were also performed to improve the performance of wind turbines. For example, a software for designing the optimal shape of multi-MW wind turbine blades and analyzing the performance was completed, and it featured aerodynamic shape design, performance analysis, pitchtorque analysis and shape optimization for wind turbine blades (Kim et al. 2013).

Those investigations greatly improved the performance of a modern wind turbine. However, there are some concerns on the aerodynamic noise, which is generated from a wind power plant, for the technicians who work in close proximity to the wind turbine (Hubbard and Shepherd 1991; Giguere and Selig 1997; Hansen 2007; Van den Berg 2008; Jaskelevicius and Užpelkiene 2008; Genescà et al. 2009; Watson et al. 2010; Lee et al. 2011). A local resident 
has the right to live quietly within the limits of the environmental standards (Lercher 1996; Japanese-Government 1998; Waye and Ohrstrom 2002). Therefore, maintaining the working environment quietly is especially important for health maintenance of the technicians who work in close proximity to the wind turbine. This is a problem which cannot be bypassed when conquering an environmental problem.

In general, there are mainly two potential sources of noise generated by a wind power plant, a mechanical noise originated from the gear system (or gearbox) and an aerodynamic noise originated from the wind turbine. The aerodynamic noise is generally considered to be the dominant source of noise generated by a modern large-scaled wind power plant (Oerlemans et al. 2007). However, the mechanism of generating the aerodynamic noise has not been hydrodynamically elucidated yet owing to the complicated structures. In a residential area adjacent to a largescaled wind power plant, the noise emitted from the wind turbine has caused serious social and environmental problems. Moreover, such noise generated under the operation condition of a wind power plant cannot deny the possibility of causing the health impairment to a local resident, either.

Although some investigations on the aerodynamic noise have been carried out (Bjorkman 2004; Oerlemans et al. 2007; Pedersen and Waye 2004, 2007, 2008; Pedersen et al. 2009; Pedersen 2011; Zhang et al. 2012; Genescà et al. 2013), an argument about the relationship between the aerodynamic noise and the human physiological condition has not been fully settled yet. In particular, not only any mechanism of generating the infrasound noise with extremely low-frequency bands, but also any influence on human physiology has not been elucidated. As known, an electroencephalogram (EEG) examination is a method to obtain brain waves, and different brain waves frequencies show synchronies related to different perceptual, motor or cognitive states (Will and Berg 2007; Goodin et al. 2012). Therefore, the physiological measurement with EEG is carried out with respect to the aerodynamic noise generated by a large-scaled wind turbine in the present study.

The purpose of the present study is to identify the characteristics and the mechanism of the aerodynamic noise originated from a large-scaled wind turbine, and to verify the physiological impact (brain waves) affected by the aerodynamic noise and the aerodynamic sound pressure level (including infrasound with extremely low-frequency band). The brain waves, including the theta wave, the alpha wave and the beta wave, were measured with EEG study, when 15 test subjects received various sound stimuli. In the EEG study, we compared responses to recorded aerodynamic noise, periodic sound stimulations (sounds with repetition rates of 20,30,86 and $300 \mathrm{~Hz}$ ). The key point of the present study is the relationship between the aerodynamic noise and the physiological evaluation from an engineering point of view. It is believed that it is of great importance to reveal the relationship between the aerodynamic noise emitted from a wind turbine and the physiological responses (brain waves). The present study also investigated the effect of the aerodynamic infrasound noise, trying to make this research to be one of the unique investigations on the aerodynamic noise.

In order to achieve the purpose mentioned above, a series of measurements and recordings were performed around a large-scaled wind power plant, which was built at the location of above-sea-level $800 \mathrm{~m}$ on Satomi Farm in Hitachi Ota, Ibaraki, Japan, on December 8, 2010. The recorded time was totally $5 \mathrm{~min}$, from 16:33 to 16 : 38 on that day. The recording work was performed in winter in order to avoid the influence from the sound of birds and insects.

The wind turbine was made by ENERCON in Germany. The upwind type wind turbine (ENERCON E40) is equipped with three blades, which provides a round structure to reduce the aerodynamic noise. The wind turbine has the rotor diameter of $43.7 \mathrm{~m}$ and the tower height of $44.2 \mathrm{~m}$. It is a large-scaled wind turbine for a wind power plant, which was built in the collaboration with the new energy and industrial technology development organization (NEDO) and is a symbol of an environmentally friendly city plan which Hitachi Ota city advances. The capacity is about $600 \mathrm{~kW}$ as a rated electric power output. The rotational speed varies from 18.0 to $34.5 \mathrm{rpm}$, and the cut-in, cut-out and rated wind velocity are $2.5,25.0$ and $13.5 \mathrm{~m} / \mathrm{s}$, respectively. The electric power is consumed by the Satomi Farm, and the surplus electric power is sold off to an electric power company.

\section{Materials and methods}

Methods of obtaining the aerodynamic noise

During the recording of sound and sound pressure level, three kinds of measurement instruments were utilized: (a) sound recorder (Sony PCM-D50, with a sampling frequency of $44.1 \mathrm{kHz}$ and quantization of $16 \mathrm{bit}$ ), (b) sound pressure level meter (CEM DT-8852, A-weighted $(\mathrm{dB}(\mathrm{A})$ measurements), with a measurement interval of $1 \mathrm{~s}$ ) and (c) infrasound pressure level meter (RION NA-18, G-weighted $(\mathrm{dB}(\mathrm{G})$ measurements), with a measurement interval of $0.1 \mathrm{~s}$ ). Figure $1 \mathrm{a}$ represents the schematic illustration of the positions of the measurement instruments, including the sound recorder, the infrasound pressure level meter and the sound pressure level meter. These measurement instruments were installed at four positions, which were upwind side as the front side, downwind side 


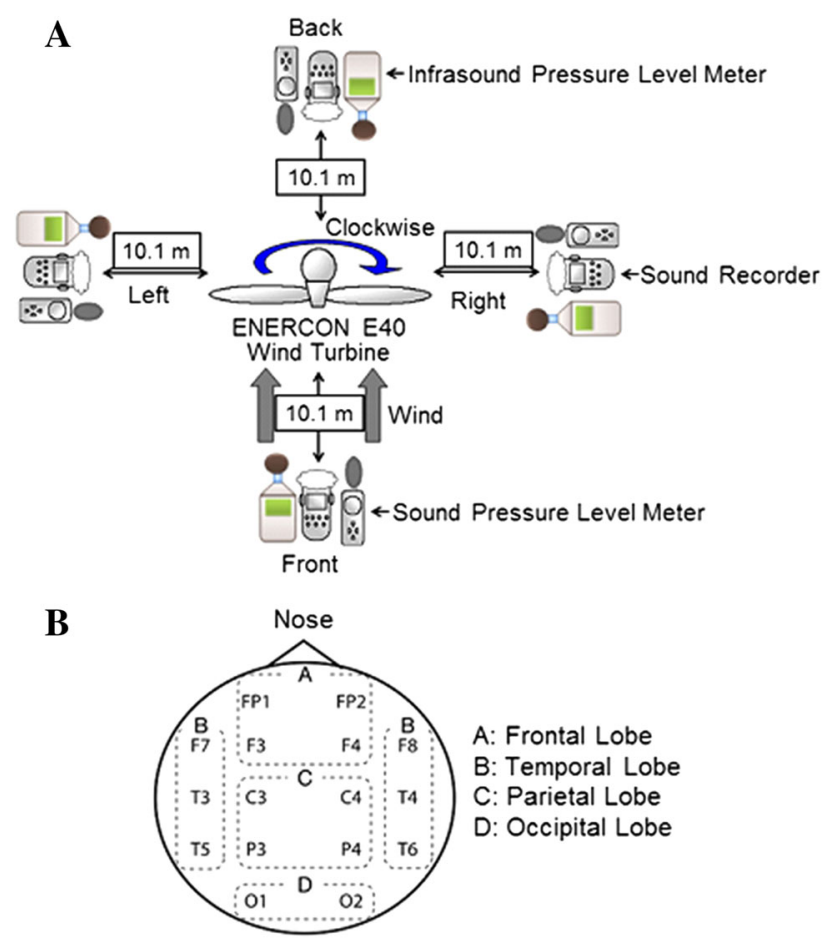

Fig. 1 Schematic illustration of the positions of a the experimental equipment and $\mathbf{b}$ the electrodes during an EEG examination

as the back side, right-hand side and left-hand side to the center of the wind turbine, to record the sound and the sound pressure level information. The distance between the three kinds of measurement instruments and the center line of the wind turbine tower was $10.1 \mathrm{~m}$ in the four directions. The image data of the electric power output, the wind direction and the wind velocity, which were recorded with a digital video camera (Panasonic TM-35) and were synchronized with these measurement instruments, were processed by a computer. A-weighting is the most commonly used curve, which is defined in the International Standard IEC 61672:2003 and various national standards relating to the measurement of sound pressure level. The unit is written as [dB (A)]. A-weighting is applied to instrumentmeasured sound pressure level so as to account for the relative loudness perceived by the human hearing, as the ears are less sensitive to relatively low audio frequencies. G-weighting is specifically designed for detecting infrasound pressure level. The recorded data were analyzed using a fast-Fourier-transform analysis (FFT) with a window type of hamming (window length of $30 \mathrm{~ms}$ ), a sonogram analysis with a window type of hamming (window length of $30 \mathrm{~ms}$ ). In addition, a wavelet analysis based on a spline function of fourth polynomial interpolation as the mother wavelet was also utilized to investigate various acoustical phenomena. Although the measurement position was influenced by the wind direction on that day, the direction of the wind turbine could not change within a short measurement time of the present study.

Methods of obtaining the brain waves

The brain waves are weak electric signals emitted from a brain when the nerve cells transmit information simultaneously. The brain waves exhibit the cerebral activities and the level of the consciousness conditions. When a brain is active, various electric signals overlap each other and the brain waves become a fine waveform at random as a whole. On the other hand, when a brain is not active, various electric signals are synchronized to some extent; thus, the brain waves become a calm waveform. In general, the brain waves are classified into four zones, according to the frequency band after applying FFT analysis, and are named as the delta wave ( $\delta$ rhythm), which represents a deep sleep state, the theta wave ( $\theta$ rhythm, with the frequency range from 4 to $8 \mathrm{~Hz}$ ), which expresses a doze state (light sleepy state with dreaming and decreasing of awareness level), the alpha wave $\left(\alpha_{1}, \alpha_{2}\right.$ rhythms, with the frequency range from 8 to $10 \mathrm{~Hz}$ and range from 10 to $13 \mathrm{~Hz}$, respectively), which indicates a relaxed and concentrated state (low level
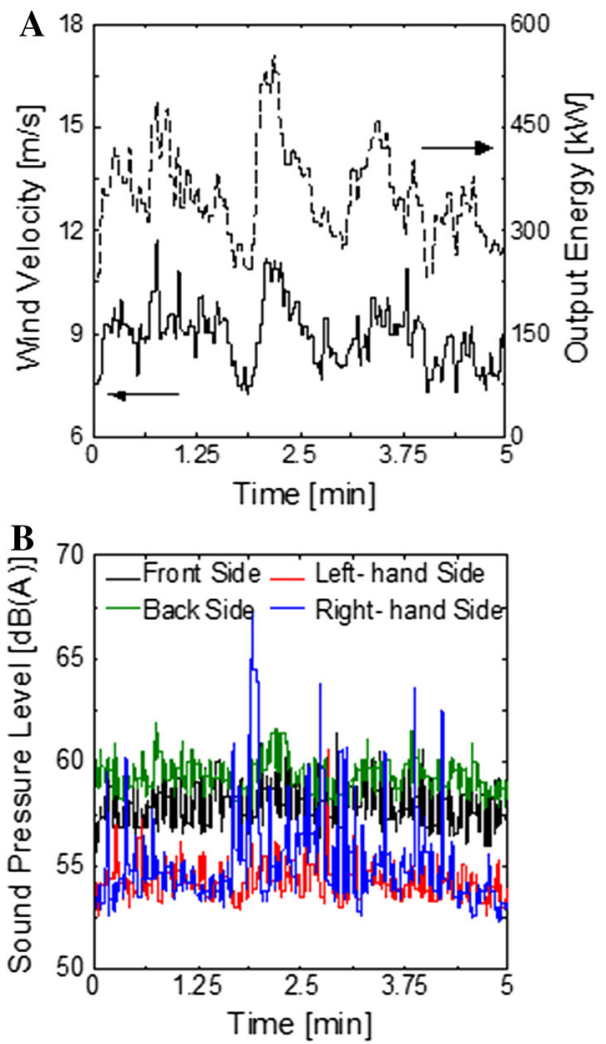

Fig. 2 Time-series data of a the wind velocity and the output energy; b the aerodynamic sound pressure level (A-weighting) at four measurement points 
Fig. 3 Frequency

characteristics included in four

time-series data of the

aerodynamic sound noise at four

measurement points
A

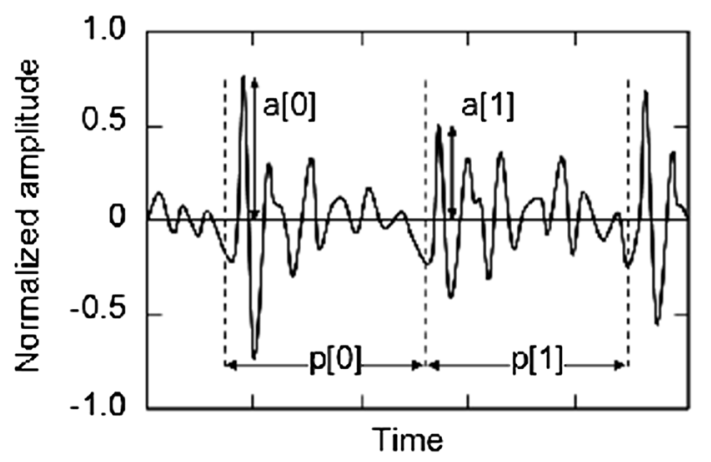

B
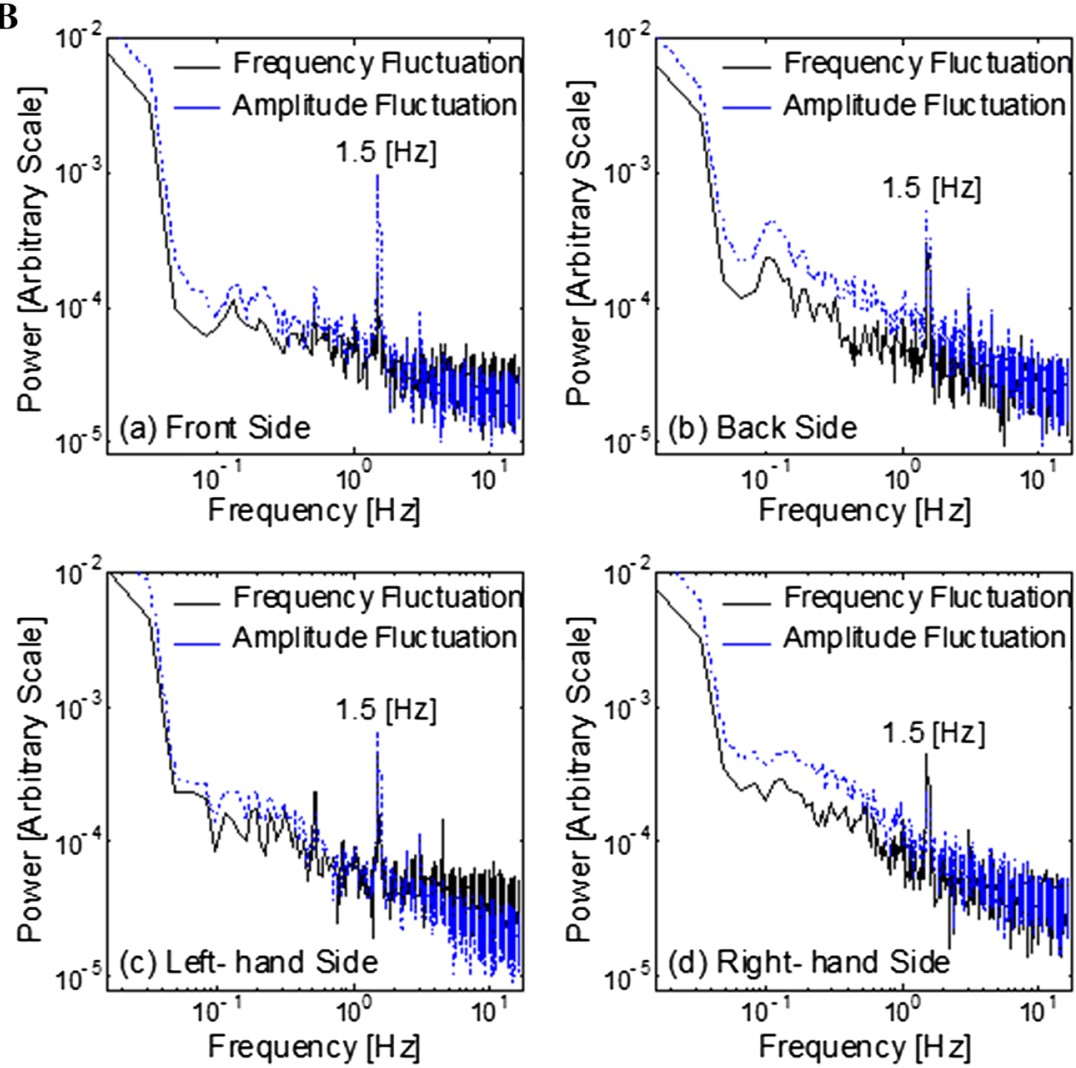

and high level, respectively), and the beta wave $\left(\beta_{1}, \beta_{2}\right.$ rhythms, with the frequency range from 13 to $20 \mathrm{~Hz}$ and range from 20 to $35 \mathrm{~Hz}$, respectively), which shows a strain state (with clear consciousness and with unclear consciousness, respectively).

In order to elucidate the effect of aerodynamic noise on human physiology, i.e., human perceptions responsive to aerodynamic noise, which was generated by a large-scaled wind turbine, the changing of brain waves was investigated by means of the EEG measurement. The brain waves were recorded with an EEG (Nihon Kohden EEG9100) using a both earlobes connecting method, which is an international standard when receiving various stimuli of aerodynamic noise. Figure $1 \mathrm{~b}$ represents the positions of the electrodes and the locations of the lobes during the EEG measurement. Electrodes are installed in 16 places on the scalp corresponding to the frontal lobe, the parietal lobe, the occipital lobe of cerebrum and the temporal lobe on both sides. The sampling frequency of the EEG examination is $200 \mathrm{~Hz}$, and the sensitivity is $10 \mu \mathrm{V} / \mathrm{mm}$. The test subjects are fifteen healthy adults (21-24 years old, with the average age of 23 years old).

One of the sound stimuli used for measuring the brain waves was the aerodynamic noise, which was actually recorded outdoors near the large-scaled wind turbine, and the sound pressure level was artificially set at $50 \mathrm{~dB}$ (A) or $55 \mathrm{~dB}$ (A). Another sound stimulus, which was composed of four periodical sounds with frequency bands of $20,30,86$ and $300 \mathrm{~Hz}$, was also artificially synthesized to make a comparison with the aerodynamic noise, and the sound pressure level was artificially set at $50 \mathrm{~dB}(\mathrm{~A})$ or $55 \mathrm{~dB}(\mathrm{~A})$, either. Each wave shape of the 
Fig. 4 Results of the FFT analysis based on the timeseries data of the aerodynamic sound noise at four measurement points
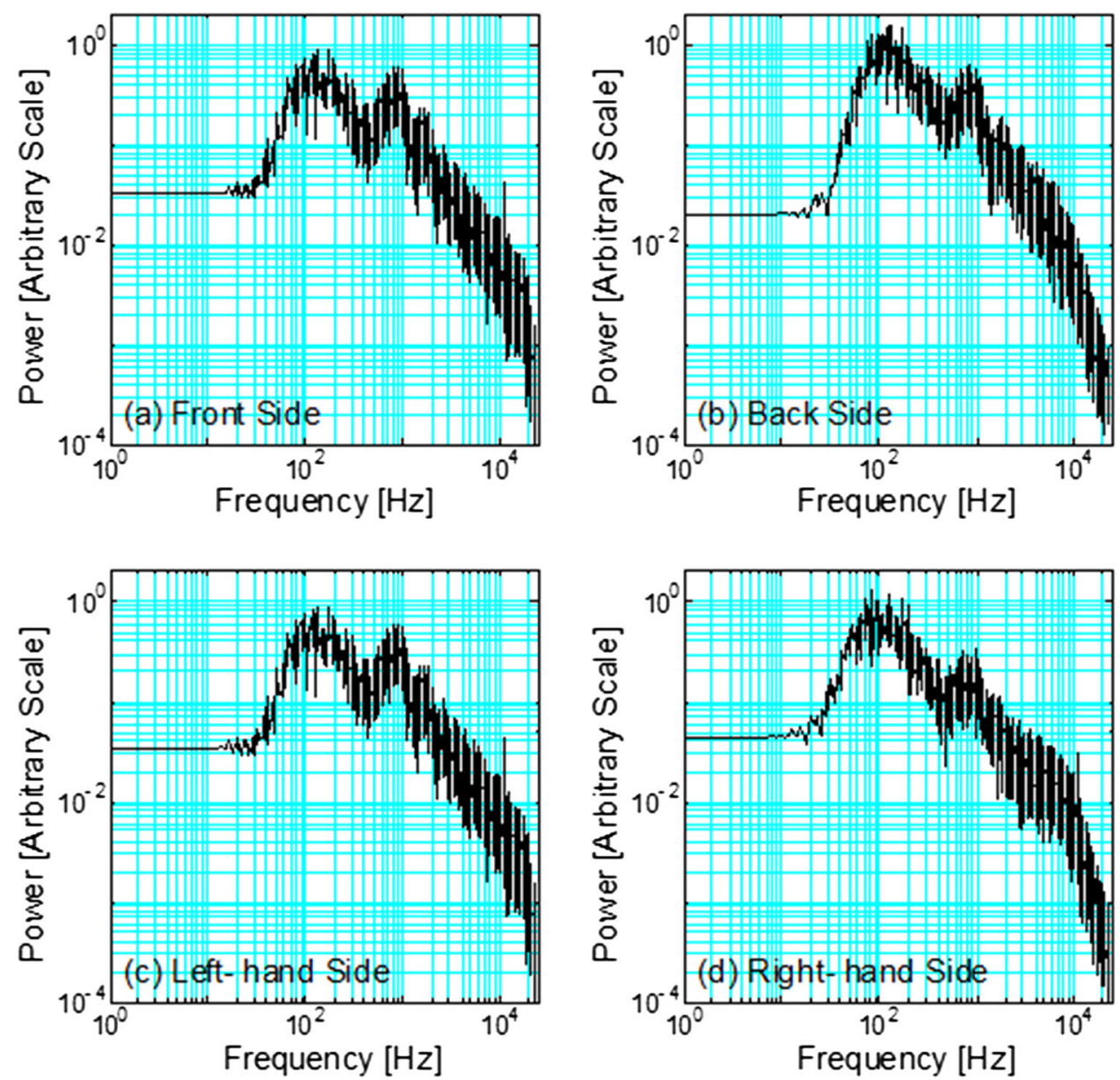

artificial periodical sound was composed of a beat cycle with $1 \mathrm{~s}$. The EEG measurement was performed in a shield room to maintain a darkness condition. During the examination, a test subject was asked to close eyes and sit quietly and comfortably in front of two speakers (VICTOR UX-A5MD, with the frequency response range of 200-20,000 Hz) and a super woofer (VICTOR SX-DW77, with the frequency response range of 16-250 Hz). The distance between the test subject and the sound devices was set at $1.3 \mathrm{~m}$. A test subject, equipped with the EEG instruments, was asked to hear five kinds of sound stimuli for $120 \mathrm{~s}$ and followed by a silent state for $60 \mathrm{~s}$. The order of giving five kinds of sound stimuli was random.

In the present study, the area ratio, in which each frequency band of brain waves $\left(\alpha_{1}, \alpha_{2}, \beta_{1}, \beta_{2}, \theta\right)$ occupies, was defined as the occupied rate. The induced rate was defined as the ratio between the occupied rate of brain waves during the sound stimuli and the occupied rate of brain waves when closing eyes under quiet state. Delta wave is not considered for a physiological evaluation, because the delta wave shows a deep sleep state on the physiological consideration. The other brain waves (theta, alpha and beta waves) are set as the objects during the physiological evaluation.

Occupied rate $=\frac{\text { area, in which each frequency band of brain waves }\left(\alpha_{1}, \alpha_{2}, \beta_{1}, \beta_{2}, \theta\right) \text { occupies }}{\text { area which all the frequency bands of brain waves occupy }}$

Induced rate $=\frac{\text { each occupied rate of brain waves }\left(\alpha_{1}, \alpha_{2}, \beta_{1}, \beta_{2}, \theta\right) \text { during stimuli by some sound noises }}{\text { each occupied rate of brain waves }\left(\alpha_{1}, \alpha_{2} \beta_{1}, \beta_{2}, \theta\right) \text { when closing eyes under quiet state }}$ 
A Date: $8^{\text {th }}$ December 2010 Recording time: 16:33-16:38 (5 [min])

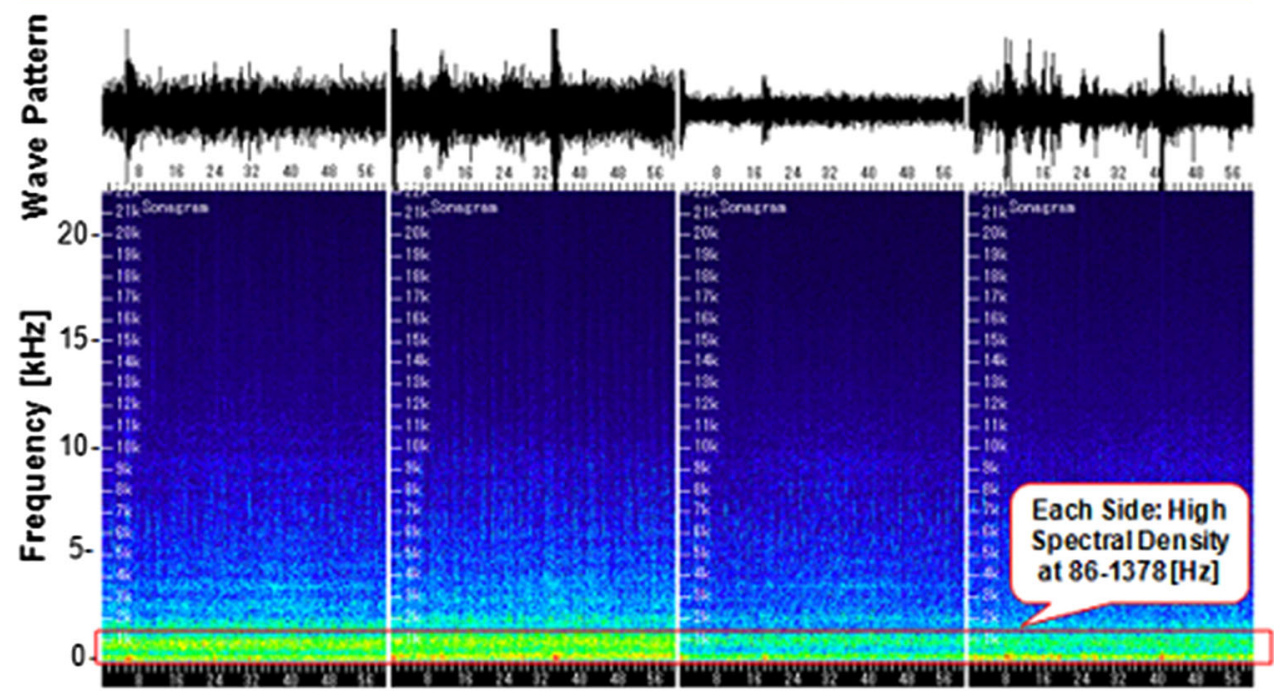

Time [s]

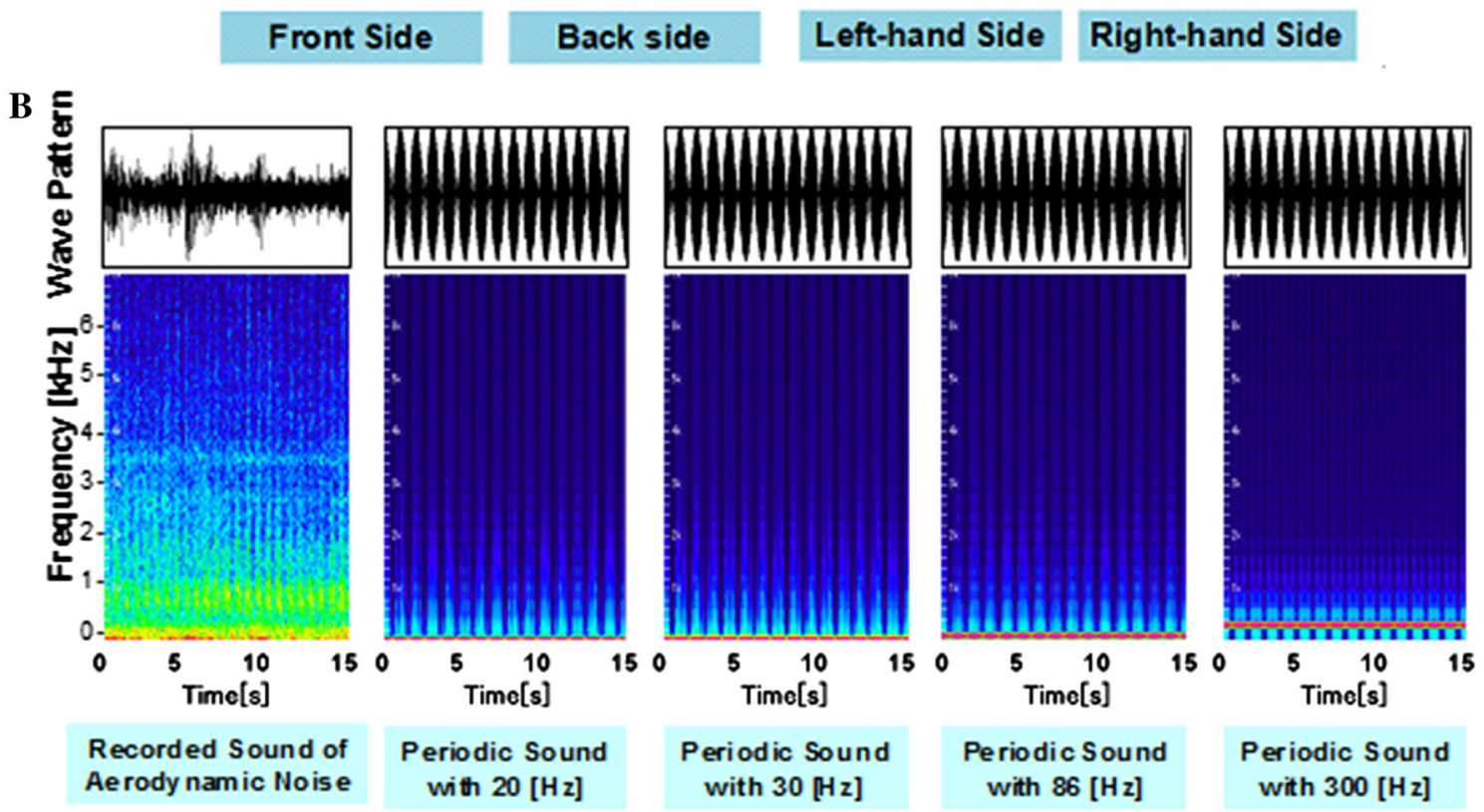

Fig. 5 Results of the sonogram analysis based on a the time-series data of the aerodynamic sound noise at four measurement points $\mathbf{b}$ the synthesized sound stimuli

The induced rate is a physical quantity which shows the rate of brain waves, which was induced in response to a certain sound stimulus, and expresses the physiological reaction of a test subject to the sound stimuli. The physiology state, which a test subject receives, becomes clear by analyzing the induced rate of brain waves. The induced rate is therefore a very important index in evaluating the physiological state of a person, who lives in the area close to a large-scaled wind power plant. A $\chi^{2}$-test is also applied to the induced rate of brain waves in the present investigation, to statistically eliminate the unreliable data in the EEG information.

\section{Results and discussion}

Results of aerodynamic noise

Figure 2a presents the time-series data of the wind velocity (solid line) measured with an anemometer installed at the upper part nacelle of the large-scaled wind turbine for 5 min (16:33-16:38) on 8th December, 2010. The transient electric output energy (electric power supply) is illustrated by the dash line. The experimental condition at that time: wind blows from the northwest at an average velocity of $8.9 \mathrm{~m} / \mathrm{s}$, and the average electric output was $353 \mathrm{~kW}$. 
Fig. 6 Results of the wavelet analysis based on the timeseries data of the aerodynamic sound noise at four measurement points
Date: $8^{\text {th }}$ December 2010 Recording time: 16:33-16:38 (5 [min])

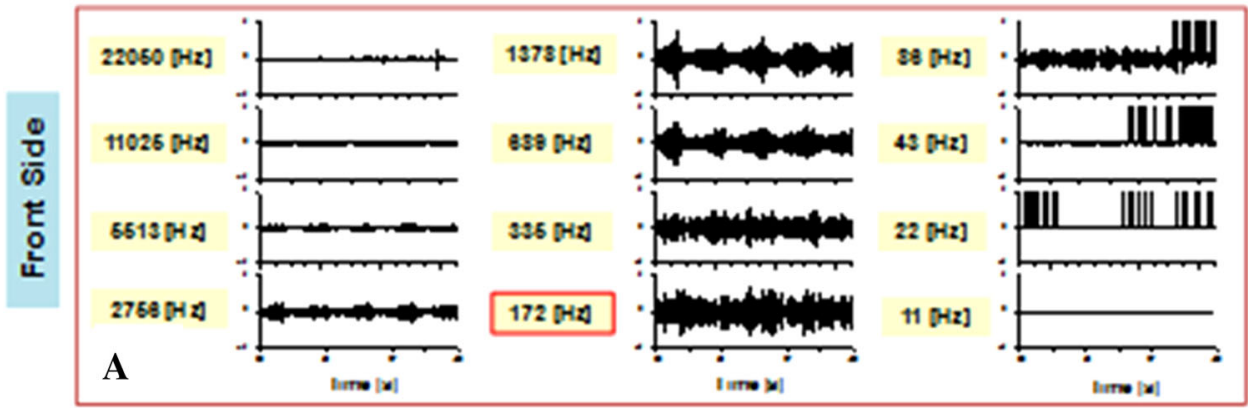

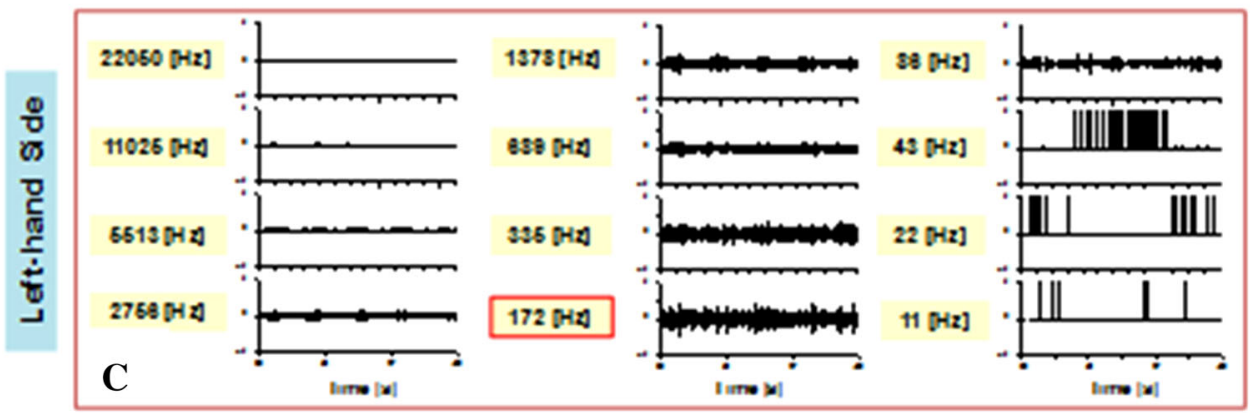

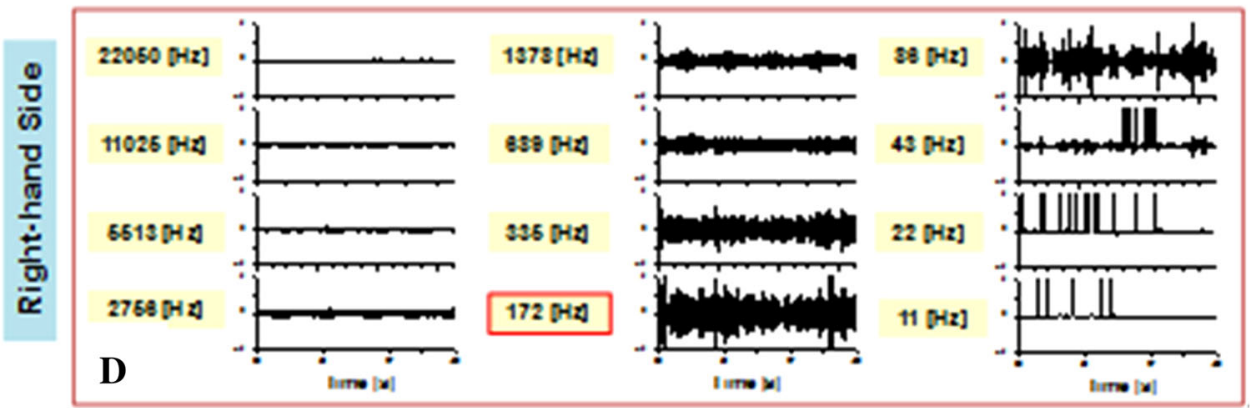

Figure $2 \mathrm{~b}$ shows the time-series data of the aerodynamic sound pressure level (A-weighting) measured with sound pressure level meters at four measurement points, as shown in Fig. 1a. These time-series data are synchronized with the data shown in Fig. 2a. The average sound pressure levels are $58.0 \mathrm{~dB}(\mathrm{~A})$ at front side, $59.3 \mathrm{~dB}(\mathrm{~A})$ at back side, $54.3 \mathrm{~dB}(\mathrm{~A})$ at left-hand side and $55.2 \mathrm{~dB}$ (A) at righthand side. It turns out that the sound pressure level differs from each other at the four measurement points. It is also seen that the pressure level detected by the sensors installed at the front and the back sides are larger than those obtained at the left-hand and the right-hand sides. The value of the sound pressure level at the back side is the largest.

According to the environmental standards of the sound pressure level (Japanese-Government 1998), the noise limit for residential area is $55 \mathrm{~dB}(\mathrm{~A})$ during daytime (6 a.m. to 10 p.m.) and $45 \mathrm{~dB}$ (A) during nighttime (10 p.m. to 6 a.m.). In addition, the noise limit for rural area and mixed (residential, commercial and industrial) area are $50 \mathrm{~dB}(\mathrm{~A})$ and $60 \mathrm{~dB}$ 


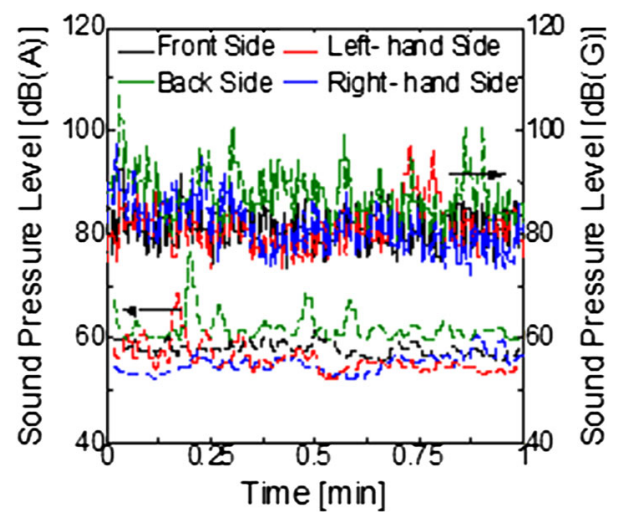

Fig. 7 Time-series data of the sound pressure level (A-weighting and G-weighting) at four measurement points

(A) during daytime, and $40 \mathrm{~dB}(\mathrm{~A})$ and $50 \mathrm{~dB}(\mathrm{~A})$ during nighttime, respectively. From Fig. 2b, it can be seen that the sound pressure level typically measured in decibels extremely near the large-scaled wind turbine is over the noise limits defined by the environmental standards.
There is a possibility that the infrasound can be also an annoyance to the technicians who work in close proximity to the wind turbine. However, the actual residential section is located at the place far from a wind power plant compared with the present case, and the sound pressure level may become much smaller.

At first, we estimated the mechanical characteristic of the present wind turbine, aerodynamic movement of the blades such as rotation rate. Figure $3 \mathrm{a}$ represents a concept model of determining frequency fluctuation and amplitude fluctuation included in the time-series aerodynamic noise data, which were obtained with the sound recorder. The amplitude fluctuation a[i] represents the sound strength, which is derived from the maximum value in a unit time $\mathrm{p}[\mathrm{i}]$, where $\mathrm{p}[\mathrm{i}]$ represents the time between two successive amplitude fluctuation $(\mathrm{a}[\mathrm{i}])$. In addition, the frequency fluctuation represents the sound height, which is defined as the number of zero-crossing points in a unit time p[i]. Figure $3 b$ shows the frequency characteristics, calculated with FFT analysis method. It is based on the frequency fluctuation and amplitude fluctuation derived from the four

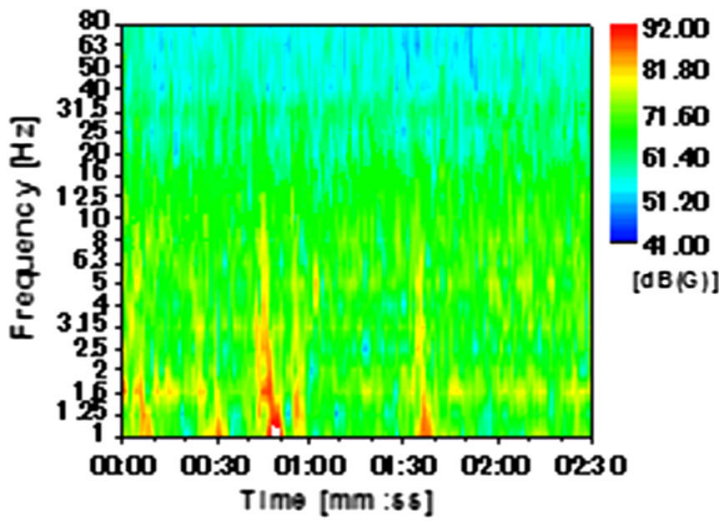

A Front Side

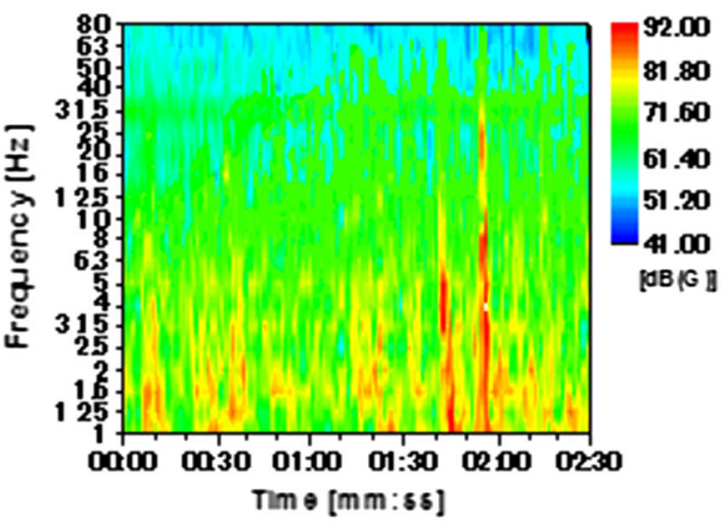

C Left-hand Side

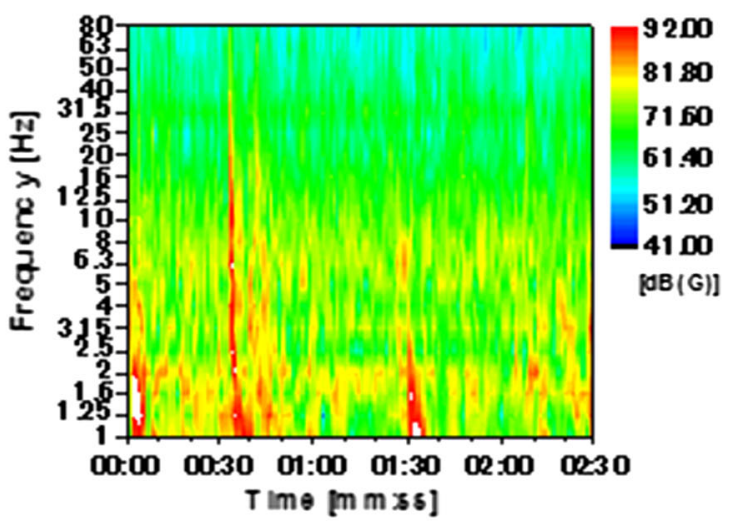

B back Side

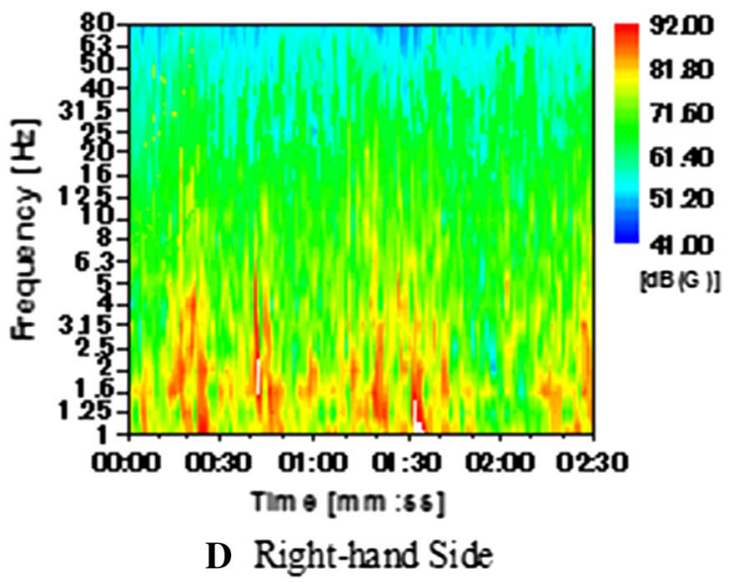

Fig. 8 Results of the sonogram analysis based on time-series data of the infrasound pressure level at four measurement points 


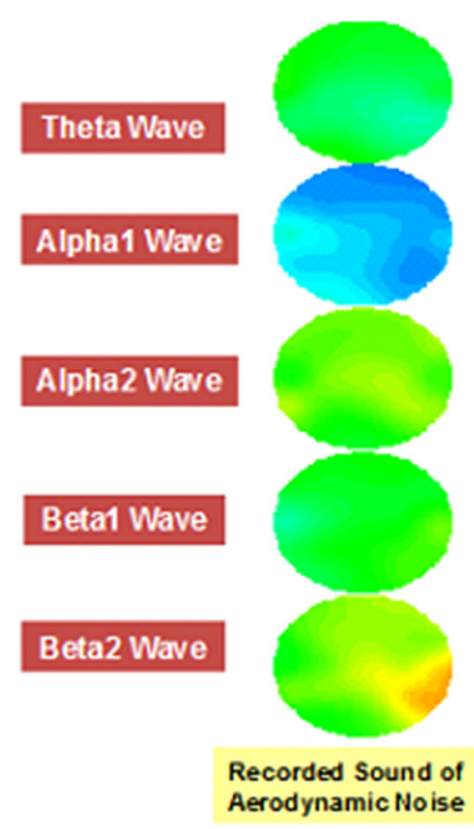

A

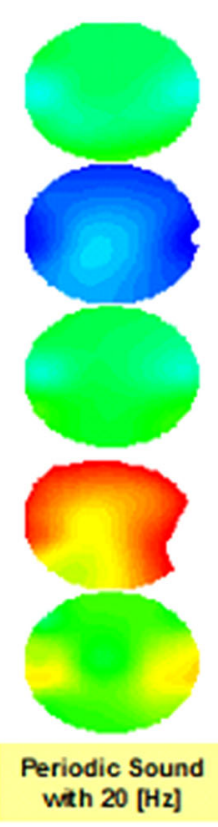

B

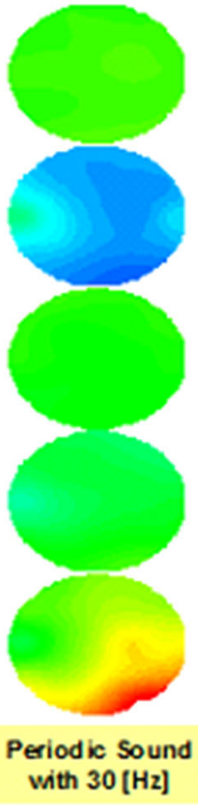

C

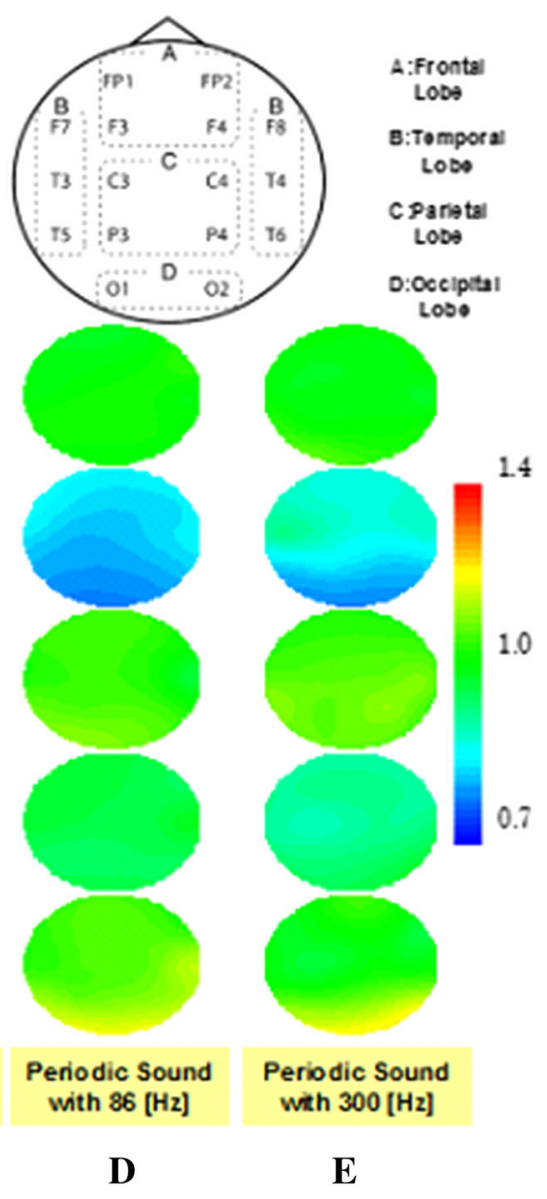

Fig. 9 Mapping patterns of the averaged induced rate of the brain waves among the test subjects

time-series data of the aerodynamic noise, which was measured with four sound recorders at four measurement points shown in Fig. 1a. It turns out that the frequency characteristics of the aerodynamic noise are similar at four measurement points, and there is a peak value at $1.5 \mathrm{~Hz}$ at all measurement points. This means that the time for one blade of wind turbine to pass through the support is $0.67 \mathrm{~s}$, and the rotation rate of the wind turbine can be determined as $30 \mathrm{rpm}$ from the measured time interval.

Figures 4 and 5a show the results of the FFT analysis and the sonogram analysis. The results are calculated from the four time-series data of the aerodynamic noise that was measured with the sound recorders at four measurement points shown in Fig. 1a. The upper graphs in Fig. 5a show the time-series wave pattern of the aerodynamic noise and the lower graphs show the sonogram analysis results. It is observed from Fig. 4 that there are three peaks in the frequency band ranged from 80 to $2,000 \mathrm{~Hz}$. It is also found from Fig. 5a that the spectral density becomes large in the same frequency range and the sound wave differs from each other at four measurement points.
Figure 6 shows the results of the wavelet analysis based on four time-series data of the aerodynamic noise. It is observed that the value of the aerodynamic noise at the back side is the largest among the other positions. In particular, the value is the largest at the frequency band of $172 \mathrm{~Hz}$, which is consistent with the previous results of the sonogram analysis shown in Fig. 5a. It turns out that the sound wave differs from each other at four measurement points and the spectral density calculated from the timeseries aerodynamic noise data extracted at four measurement points becomes large at the frequency bands of 86-1,378 Hz. It is therefore assumed that the aerodynamic noise generated by the large-scaled wind turbine is composed of low-frequency noise and high-frequency noise.

Figure 7 expresses the time-series data of A-weighting and G-weighting sound pressure level measured with the sound pressure level meters and the infrasound pressure level meters for 1 min (16:33-16:34) on December 8, 2010, at four measurement points. It turns out that the infrasound pressure level differs from each other at four measurement points. It is found that the value of infrasound pressure level detected by the infrasound pressure level 

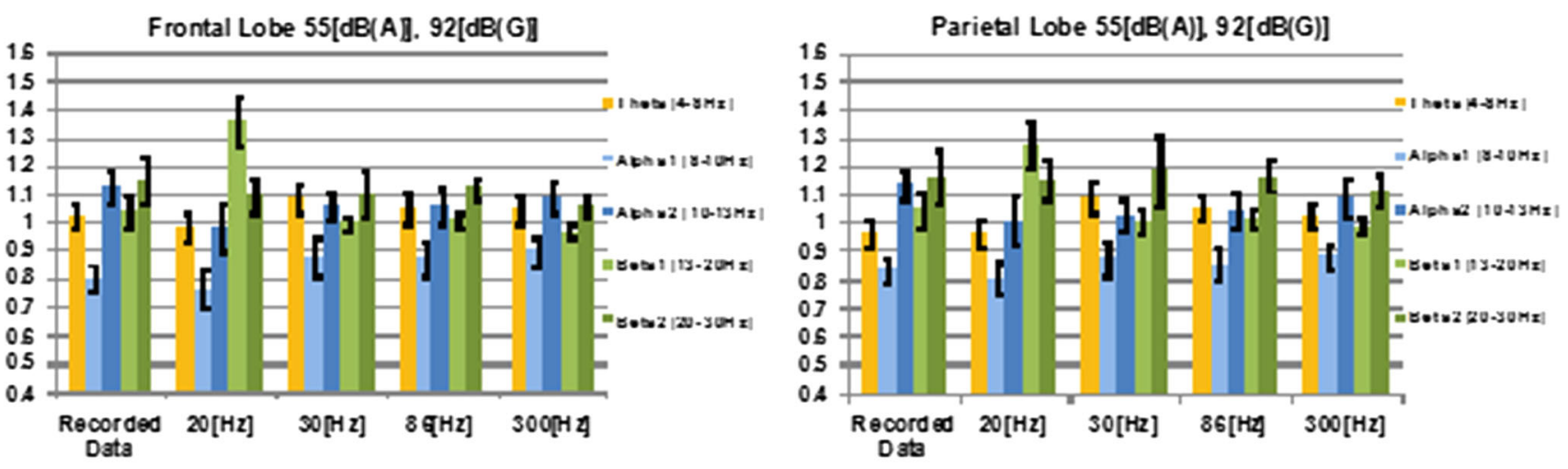

L-Tem poral Lobe 55[dB(A)]. 92[dB(G)]
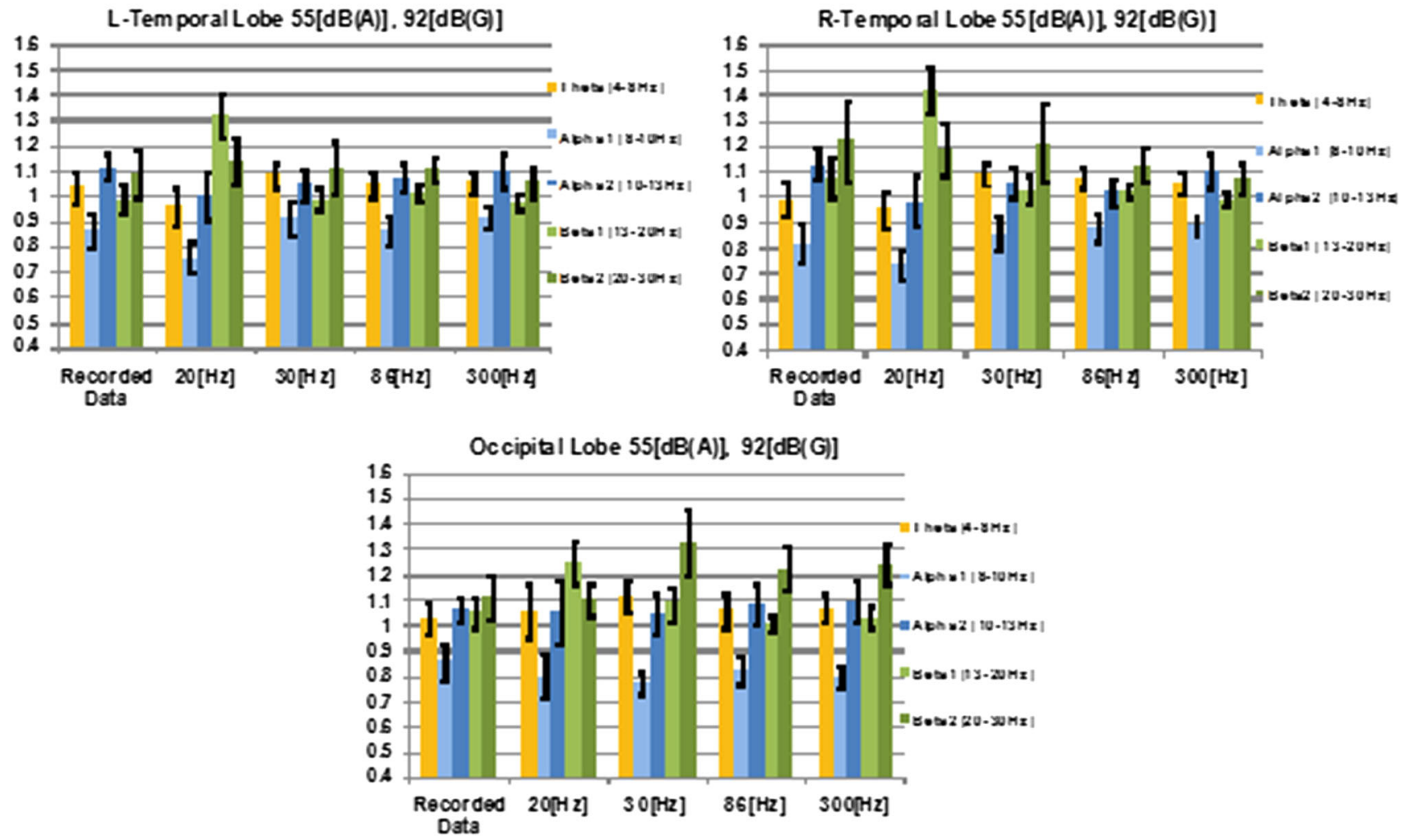

Fig. 10 Averaged induced rate of the brain waves with its standard error

meters installed at the front and the back sides are larger than those at the left-hand and the right-hand sides. The value of the infrasound pressure level at the back side seems to be the largest among the other data, because the induced aerodynamic noise is being carried with the wind. Figure 8 shows the results of the sonogram analysis based on four time-series data of the infrasound pressure level, which is measured with the infrasound pressure level meters at four measurement points. It is observed that the spectral density calculated from the time-series data extracted at four measurement points is large at the frequency bands of $1-10 \mathrm{~Hz}$, which occurs around the support of the wind turbine (wind turbine tower) by Karman vortex and cannot be detected by human hearing.
As mentioned above, the aerodynamic noise is always required to be considered carefully in the noise generated by a large-scaled wind turbine. It is found in our work that the aerodynamic noise is composed of high-frequency noise and low-frequency noise including infrasound noise. The former could be originated from an eddy emission, generated along the blades of wind turbine, and the latter could be originated from a flow phenomenon, generated around the support of wind turbine (wind turbine tower). For instance, since a certain amount of sound wave goes up to the frequency of $1,378 \mathrm{~Hz}$ is obvious continuously, as shown in Fig. 6, the noise of these frequency bands may radiate from the trailing edge, which is caused by the turbulent eddies passing the trailing edges of the blades. 
Moreover, since a certain amount of the aerodynamic noise is large in the frequency band of $86-335 \mathrm{~Hz}$, it turns out that the noise of this frequency band corresponds to the low-frequency noise, which is caused by the tower wake passing the tower cross section. Therefore, it can be concluded that the aerodynamic noise generated by the largescaled wind turbine is composed of low-frequency noise and trailing edge noise.

\section{Results of brain waves}

Figure $5 b$ presents the testing frequency and the wave patterns of the sound stimuli for EEG examinations, whereby the sound recorded near the large-scaled wind turbine and the synthesized periodical sound with the frequency band of 20,30, 86 and $300 \mathrm{~Hz}$, respectively. One of the sound stimuli used for measuring the brain waves (EEG) is the aerodynamic noise, which was actually recorded outdoors near the large-scaled wind turbine, with the sound pressure level artificially set at $50 \mathrm{~dB}$ (A) or $55 \mathrm{~dB}$ (A). Another sound stimulus, which is composed of four periodical sound waves with the frequency bands of 20,30, 86 and $300 \mathrm{~Hz}$, is also artificially synthesized to compare with the aerodynamic noise, while the sound pressure level was artificially set at $50 \mathrm{~dB}$ (A) or $55 \mathrm{~dB}$ (A).

Figure 9 illustrates the mapping pattern of the EEG examinations when the test subjects are listening to the aerodynamic noise generated by the large-scaled wind turbine and the above-mentioned four other kinds of periodical sound waves with a sound pressure level of $55 \mathrm{~dB}$ (A). The mapping pattern indicates the averaged induced rate of brain waves $\left(\theta\right.$ rhythm, $\alpha_{1}$ rhythm, $\alpha_{2}$ rhythm, $\beta_{1}$ rhythm and $\beta_{2}$ rhythm). It is seen that $\alpha_{1}$ rhythm, after the sound stimulus with the frequency band of $20 \mathrm{~Hz}$, is the lowest among others. Meanwhile, $\beta_{1}$ rhythm, after the sound stimulus with the frequency band of $20 \mathrm{~Hz}$, is the highest.

Figure 10 presents the averaged induced rate of the brain waves between 15 test subjects and its standard error, which is illustrated by the black error bar on the top of each column. The induced rate of $\theta$ rhythm has almost no change after the sound stimuli, but the induced rate of $\alpha_{1}$ rhythm becomes lower when the test subjects listen to all the sound and decreases with decreased frequency band. In particular, the induced rate of $\alpha_{1}$ rhythm after the sound stimulus with the frequency band of $20 \mathrm{~Hz}$ becomes the lowest among the other cases. This means that the test subjects cannot be relaxed comfortably when listening to the low-frequency noise or the infrasound noise, which is a part of the aerodynamic noise generated by the large-scaled wind turbine. In addition, a similar variation tendency of the brain waves was observed when the sound pressure level was artificially set at $50 \mathrm{~dB}(\mathrm{~A})$.

\section{Conclusion}

In the present study, the aerodynamic noise generated by a modern large-scaled wind power plant was measured and analyzed from an engineering point of view. The measurement items were the sound, the sound pressure (noise level) including the infrasound with extremely low-frequency wave bands and the corresponding physiological responses (brain waves). The brain waves were measured with EEG examination when the test subjects received various stimuli of sound. This research evaluated the relationship between the infrasound with extremely lowfrequency band and the brain waves. It is noteworthy that our work verified the influence of aerodynamic noise on the brain waves for the first time.

The infrasound emitted from the large-scaled wind turbine could be an annoyance to the technicians who work in close proximity to the wind turbine. The sound pressure level at the front and the back sides are larger than those at the lefthand and the right-hand sides of the large-scaled wind turbine. There is a peak value of $1.5 \mathrm{~Hz}$ in the sound wave frequency spectrum for each measurement point, which means the time for one blade of the wind turbine to pass through the support was $0.67 \mathrm{~s}$ (rotation rate $30 \mathrm{rpm}$ ). The spectral density calculated from the time-series data of the aerodynamic noise extracted at four measurement points is large at the frequency bands of $86-1,378 \mathrm{~Hz}$. It is found that the aerodynamic noise generated by the large-scaled wind turbine is composed of low-frequency noise and trailing edge noise. The induced rate of alpha $a_{1}$ rhythm decreases when the test subjects listen to all the sound stimuli and further decreases with decreased frequency. In particular, the induced rate of alpha $a_{1}$ rhythm, after the sound stimulus at the frequency band of $20 \mathrm{~Hz}$, is the lowest value among the other cases. This means that the subjects cannot be relaxed comfortably when listening to the infrasound noise.

Acknowledgments The authors want to present thanks to Mr. Kei Miyauchi, Ms. Nur Akmal Binti Haniffah and Ms. Airi Watanabe for helping to gather relevant information.

\section{References}

Baath LB (2013) Noise spectra from wind turbines. Renew Energy 57:512-519

Bjorkman M (2004) Long time measurements of noise from wind turbines. J Sound Vib 277:567-572

Chen J, Wang Q, Shen WZ, Pang X, Li S, Guo X (2013) Structural optimization study of composite wind turbine blade. Mater Des 46:247-255

Genescà M, Romeu J, Pàmies T, Sánchez A (2009) Real time aircraft fly-over noise discrimination. J Sound Vib 323:112-129

Genescà M, Romeu J, Pàmies T, Solé J (2013) On the use of a linear microphone array to measure wind turbine aerodynamic noise. Wind Energy 16:65-76 
Ghoshal A, Sundaresan MJ, Schulz MJ, Pai PF (2000) Structural health monitoring techniques for wind turbine blades. J Wind Eng Ind Aerod 85:305-324

Giguere P, Selig M (1997) Low Reynolds number airfoils for small horizontal axis wind turbines. Wind Energy 21(6):367-380

Göçmen T, Özerdem B (2012) Airfoil optimization for noise emission problem and aerodynamic performance criterion on small scale wind turbines. Energy 46:62-71

Goodin P, Ciorciari J, Baker K, Carey A-M, Harper M, Kaufman J (2012) A high-density EEG investigation into steady state binaural beat stimulation. PLoS ONE 7(4):e34789. doi:10.1371/ journal.pone.0034789

Hansen M (2007) Aeroelastic instability problems for wind turbines. Wind Energy 10:551-577

Hubbard HH, Shepherd KP (1991) Aeroacoustics of large wind turbines. J Acoust Soc Am 89:2495-2508

Japanese-Government (1998) Ministry of the environment (government of japan). Environmental quality standards for noise. Environment Agency Notification, 64

Jaskelevicius B, Užpelkiene N (2008) Research and assessment of wind turbine's noise in Vydmantai. J Environ Eng Landsc Manag 16:76-82

Jowder FAL (2009) Wind power analysis and site matching of wind turbine generators in Kingdom of Bahrain. Appl Energy $86: 538-545$

Kim B, Kim W, Lee S, Bae S, Lee Y (2013) Development and verification of a performance based optimal design software for wind turbine blades. Renew Energy 54:166-172

Lee S, Kim K, Choi W, Lee S (2011) Annoyance caused by amplitude modulation of wind turbine noise. Noise Control Eng J 59:38-46

Lee J, Son E, Hwang B, Lee S (2013a) Blade pitch angle control for aerodynamic performance optimization of a wind farm. Renew Energy 54:124-127

Lee S, Lee S, Ryi J, Choi JS (2013b) Design optimization of wind turbine blades for reduction of airfoil self-noise. J Mech Sci Technol 27(2):413-418

Lercher P (1996) Environmental noise and health: an integrated research perspective. Environ Int 22:117-128

Malhotra P, Hyers RW, Manwell JF, McGowan JG (2012) A review and design study of blade testing systems for utility-scale wind turbines. Renew Sustain Energy Rev 16:284-292

Nagai BM, Ameku K, Roy JN (2009) Performance of a 3 kw wind turbine generator with variable pitch control system. Appl Energy 86:1774-1779
Oerlemans S, Sijtsma P, López BM (2007) Location and quantification of noise sources on a wind turbine. J Sound Vib 299:869-883

Pedersen E (2011) Health aspects associated with wind turbine noiseresults from three field studies. Noise Control Eng J 59:47-53

Pedersen E, Waye KP (2004) Perception and annoyance due to wind turbine noise-a dose-response relationship. J Acoust Soc Am 116:3460-3470

Pedersen E, Waye KP (2007) Wind turbine noise, annoyance and selfreported health and well-being in different living environments. J Occup Environ Med 64:480-486

Pedersen E, Waye KP (2008) Wind turbines-low level noise sources interfering with restoration? Environ Res Lett 3(1):1-5

Pedersen E, Berg F, Bakker R (2009) Response to noise from modern wind farms in the Netherlands. $J$ Acoust Soc Am 126(2):634-643

Pratumnopharat P, Leung PS, Court RS (2013) Extracting fatigue damage parts from the stress-time history of horizontal axis wind turbine blades. Renew Energy 58:115-126

Tascikaraoglu A, Uzunoglu M, Vural B, Erdinc O (2011) Power quality assessment of wind turbines and comparison with conventional legal regulations: a case study in turkey. Appl Energy 88:1864-1869

Van den Berg GP (2008) Wind turbine power and sound in relation to atmospheric stability. Wind Energy 11:151-169

Vick B, Broneske S (2013) Effect of blade flutter and electrical loading on small wind turbine noise. Renew Energy 50:1044-1049

Watson SJ, Xiang BJ, Yang W, Tavner PJ, Crabtree CJ (2010) Condition monitoring of the power output of wind turbine generators using wavelets. IEEE Trans Energy Conver 25:715-721

Waye PK, Ohrstrom E (2002) Psycho-acoustical characters of relevance for annoyance of wind turbine noise. J Sound Vib 250:65-71

Will U, Berg E (2007) Brain wave synchronization and entrainment to periodic acoustic stimuli. Neurosci Lett 424:55-60

Yang B, Sun D (2013) Testing, inspecting and monitoring technologies for wind turbine blades: a survey. Renew Sustain Energy Rev 22:515-526

Yen J, Ahmed NA (2013) Enhancing vertical axis wind turbine by dynamic stall control using synthetic jets. J Wind Eng Ind Aerodyn 114:12-16

Zhang P, Ding H, Le C, Liu X (2012) Test on the dynamic response of the offshore wind turbine structure with the large-scale bucket foundation. Proc Environ Sci 12:856-863 